\title{
Out of hours intravenous fluid therapy: a prompt to guide prescribing
}

\author{
Janev Fehmi, Alexander Carpenter, Matthew Townsend, Rhian Sheppeard, Elizabeth Gannon, Lynsey Hewitson, Natalie Auerbach, Katherine \\ Thomas
}

University Hospitals Bristol, United Kingdom

\begin{abstract}
Recent NICE guidance has highlighted the importance of appropriate and safe intravenous fluid use. We aimed to improve the quality of out of hours fluid prescription in a Bristol hospital by ensuring that indications and cautions for fluid therapy were clearly documented at the time of initiation.

Time-pressured on-call doctors need quick access to information regarding patients' care. A documented "fluid plan" allows doctors to undertake a more informed assessment of the patient's fluid balance, leading to safer prescriptions.

Our ideal was for $100 \%$ of out of hours intravenous fluid prescriptions to be appropriate. Our process measures included the proportion of patients on intravenous fluids who had a documented fluid plan in the medical notes or on the prescription chart on Friday, prior to the weekend on call period. This was defined as mention of indications and/or cautions to fluid therapy.

The introduction of a sticker to prompt fluid plan documentation did marginally improve use of fluid plans. It was notable that $96 \%$ of these were followed where plans were documented $(n=23)$. Initiation of IV fluid with an accompanying plan is likely to make subsequent fluid prescriptions safer.
\end{abstract}

Rapid turnover of staff and stationary proved significant barriers to consistent implementation of the sticker. Despite these challenges we demonstrated a "proof of concept", suggesting system modification to include fluid plans is safe and effective.

\section{Problem}

A common problem encountered by on-call junior doctors at one Bristol-based teaching hospital is being asked to prescribe fluids for patients that they have no prior knowledge of. Often there is no clear plan for weekend fluid prescriptions of current inpatients and this can lead to busy on-call doctors making quick decisions about which fluids to prescribe and how quickly to give them. Inaccurate fluid prescription is potentially harmful and can cause electrolyte disturbances, pulmonary oedema and deterioration in renal function.

The aim of our project was to provide a standardised prompt to aid appropriate and safe fluid prescription. This prompt should be clearly visible on the fluids section of the trust-wide drug charts, filled in by the primary team looking after the patient and include details on indications and cautions to fluid prescription. Appropriate, personalised and visible documentation of information relevant to the ongoing prescription of fluids would thus guide subsequent prescribers, improving the safety and quality of out of hours fluid therapy.

\section{Background}

Many patients in hospital require intravenous (IV) fluid therapy. The indications are broad and include poor oral intake, excessive losses, acute kidney injury, electrolyte disturbances and emergency resuscitation among others. Fluid replacement should be tailored to individual patients, based on their need for specific volume, composition and rate of IV fluid delivery.

NICE have recently published guidelines on intravenous fluid therapy on adults in hospital [1] which aims to provide a framework for assessment, delivery and reassessment for patients receiving IV fluids and emphasised the importance of appropriate information regarding fluid prescription and review.

The National Confidential Enquiry into Perioperative Outcomes and Death (NCEPOD) 1999 [2] highlighted several deficiencies in fluid management. The prescription in elderly patients was often poor and they recommended that fluid prescription be accorded the same status as for drug prescription.

A previous quality improvement project carried out by junior doctors in another UK hospital found that introduction of a fluid prescription label to attach to the drug chart, indicating important cautions such as heart failure and chronic renal failure, improved junior doctors' confidence and safety in prescribing fluids in a busy out-of hours environment.[3] Our project aimed to take this further, incorporating other vital information across multiple PDSA cycles.

\section{Baseline measurement}

The project's end goal was for $100 \%$ of fluid prescriptions to be safe and appropriate. However, this is extremely subjective as an 
BMJ Quality Improvement Reports

outcome measure so we chose two main process measures for the sake of pragmatism and feasibility. These considered whether a fluid plan was documented for patients prescribed IV fluids, and an assessment of the whether that plan was followed if so. We also collected data on the number of patients on IV fluids, volumes of fluids prescribed, and whether interventions that we introduced were utilised. Initially we also collected other data (such as renal function and doses of intravenous diuretics) given as stat doses, as well as emergency chest $x$ rays ordered for pulmonary oedema. This was an attempt to gauge episodes of fluid overload or renal failure as balancing measures.

A sampling strategy was used, focusing specifically on two acute medical wards. We took the period of time from Friday at 17:00 till Monday 09:00 - the weekend on call period. On Monday, we collected data, including all patients who had been prescribed intravenous fluids on the Friday before the on-call period began. We collected the data described above regarding their intravenous fluid therapy. We also then examined the fluid chart and medical notes for sign of a fluid plan documented at the time of fluid prescription. We defined a fluid plan as documentation of either the indications, or, the cautions for fluid prescription. We took this liberal approach as we felt that the inclusion of either of these items of information provided contextual information which would guide subsequent prescriptions (although an ideal plan would include both). For those who had a plan, we assessed whether subsequent prescriptions adhered to, or deviated from, the plan.

On one ward, eleven sets of data were collected before any intervention was introduced. In this time 54 patients received prescriptions of IV fluids (average 2.7 litres per patient). Of these, $17 \%$ had documented fluid plans. Of those with fluid plans, $100 \%$ of these were followed.

See supplementary file: ds4722.docx - "PDSA Cycles"

\section{Design}

The aim of our project was to introduce a quick and easy method that was employable by the day team to make out of hours fluid prescribing a safer and more efficient task for doctors. This would be achieved by introducing a sticker or stamp on the fluid prescription chart to be completed by the patients' usual medical team. This communicated the purpose of and anticipated intravenous fluid prescribed, as well as any patient-specific cautions, ie heart failure. This would serve as an aid to nurses and doctors on call when assessing whether patients needed further fluids in a more timely manner without compromising patient safety.

Proving that when stickers or stamps are used they are effective, our hospital trust have shown an interest in incorporating this information into the next drug chart design in early 2015. At no extra cost this would seem sustainable and is only as dependent upon the resources currently required to produce the current drug chart. Integrating the concepts of fluid prescription into the intrinsic process (rather than as an extra step) would hopefully aid culture change, encouraging fluid prescription as an automatic step.

\section{Strategy}

PDSA cycle 1: Our first step was to begin collecting data and monitoring our process measures. We did this on the Monday morning following the weekend on-call period. After collecting baseline data for three months, we had seen a consistent trend: fluid plans were documented for less than half of patients on IV fluids. Some weeks none of the patients on fluids would have a plan. However, when a plan was documented, in all cases, this was followed. At this point we introduced our intervention: a sticker that we applied to the drug charts of patients on intravenous fluids. This sticker prompted documentation of indications and cautions to IV fluid administration, as well as whether further fluids were anticipated. Ward staff, constantly rotating, found it difficult to consistently implement the sticker - often forgetting, or not having one conveniently ready. Sticker use remained poor although the verbal feedback regarding the concept was positive. Some staff remained unaware of the sticker.

PDSA cycle 2: we realised that we would need to reiterate to the teams on the acute medical ward the importance of the form and filling it in correctly. Therefore we raised awareness by sending emails to these teams and talking to them face to face. We also publicised the project hospital wide by talking to junior staff at weekly foundation teaching sessions and both junior and senior staff at the medical grand round. Visits to the ward including the allied healthcare professionals and nursing staff attempted to explain the rationale behind the sticker. Large quantities of spare stickers were kept in clear locations on the ward. Use remained poor and feedback was received regarding the large size of the sticker - taking up multiple prescription boxes and felt to be too cumbersome.

PDSA cycle 3: the sticker design was modified, creating a smaller but more vividly coloured and visible sticker. This fit neatly into fluid prescription boxes. We also began placing the stickers on all prescription charts, on the Thursday, rather than just those already on IV fluids.

PDSA cycle 4: we identified a gap in the process - many patients were being transferred from the acute medical admission unit onto the ward being measured during the weekend. These patients were often acutely unwell and on intravenous fluids. We therefore reasoned that these patients represented a large cohort of patients who would be included in our data. We began to introduce stickers onto the medical admissions unit - onto all current inpatients. We also introduced a stamp, with the words "INDICATION:" and "CAUTIONS:" to stamp beside the fluid prescription. This allowed us to cover large volumes of blank prescription charts.

There remained challenges to our intervention. Rotating medical and nursing staff, practical limitations in ensuring the right prescription chart had a sticker at the correct time, and a reluctance to change working habits were all likely contributory factors.

However, when plans were made, they were in almost all cases, followed. This remains a significant "proof of concept" which remains a positive finding. The QIP ended at this point. Electronic 
prescribing will be introduced soon and creating an IV fluid plan will be a part of this process.

\section{Results}

Throughout the data collection period, we recorded data for 104 patients on IV fluids. Use of fluid plans remained poor on the intervention ward, only exceeding $50 \%$ of patients on IV fluids on one occasion. For many sampling periods, none of the patients had IV fluid plans.

Our intervention of a fluid prescription sticker faced significant challenges. Its use was highly variable although positive feedback was received regarding its aims and design. When used, it provided the viewer with vital necessary information for ongoing prescriptions. It is difficult to ascertain whether it had a significant effect on our main endpoint which was proportion of patients with IV fluids plans. However, this is difficult to measure due to the methodology used (run charts) and relatively low numbers of patients at each sampling period.

There remain two very interesting findings. First, that when fluid plans were documented $(n=23)$, they were followed in all but one instance. This powerful finding demonstrates that when used, fluid plans are likely to guide subsequent fluid therapy. As the team documenting the plan have improved understanding, having known the patient likely for some time, of the patient's circumstances, we can assume that this concordance of subsequent actions with the initial plan is likely to, on the whole, enhance patient safety.

Second, our control ward, a separate medical ward with no sticker intervention used, had significant fluctuations in fluid plan use, with overall better use of fluid plans than our control ward. Explanations for this may include more frequent consultant ward rounds on the control ward. Ward staff on the control ward reported thorough, holistic assessments which usually included fluid balance assessment. This may represent the influence on team / ward culture on behaviour, such as fluid balance assessment / planning, on the daily ward round.

See supplementary file: ds4721.jpg - "Run chart"

\section{Lessons and limitations}

Our main limitations were two-fold:

Measurement: In order to ensure timely (two-weekly) sampling, all members of the team undertook sampling. For the first several sampling periods, there was substantial inter-rater variability in what different observers would class as a "fluid plan". At that point we simplified our criteria to include any documented indications or cautions in an attempt to standardise further measurement.

Implementation of our intervention: this faced significant obstacles. Finding the time when ward staff are free to discuss new tools to improve care can be challenging on an acute medical ward with time and task pressures. We faced barriers with staff either un- aware of our intervention or unable to find spare stickers in a convenient manner. Fast turn-around of patients, ward paperwork and staff made consistent application of the sticker difficult.

\section{Conclusion}

There is significant variation within acute medical wards and substantial scope for improvement in documentation of fluid plans. When documented, these plans are almost invariably followed. It follows that the ability to follow guidance set by the patient's primary medical team, aware of the indications and cautions to intravenous fluid therapy, will enhance the appropriateness and safety of subsequent fluid prescriptions.

In principle, the idea of incorporating such information into the standardised fluid prescription chart, visible at the point of prescription for subsequent attending doctors, was felt to minimise extra work by on-call doctors and reduce the potential for error, but:

The real-life introduction of interventions such as fluid plan stickers, on an acute medical ward with rapid turnover of staff, patients, and ward paperwork, presents significant challenges to effective implementation and use of such interventions. We recorded variable response to such interventions, which were in the main part related to either non-use or non-availability of the intervention.

Systematic incorporation of fluid planning either via re-design of the fluid prescription chart, or incorporation into online fluid-prescribing may provide a more robust tool for ensuring appropriate handover to on-call doctors.

\section{References}

1. National Institute for Health and Care Excellence. Intravenous fluid therapy in adults in hospital. NICE. 2013. Available from: http://www.nice.org.uk/guidance/cg174

2. National Confidential Enquiry into Patient Outcome and Death. Extremes of Age. NCEPOD. 1999. Available from: http://www.ncepod.org.uk/1999ea.htm

3. Hartridge-Lambert S, Moore S, Walker O, Wilkinson D. Improving out-of-hours intravenous fluid prescribing for junior doctors: a prescription label. BMJ Qual Improv Rep 2013;2.

\section{Declaration of interests}

There are no conflicts of interest or funding sources to declare.

\section{Acknowledgements}

Many thanks to those within our hospital's junior doctor's quality improvement group and to those within Trust management teams for their ongoing support throughout the project. 